\title{
Relación entre el uso de glucocorticoides y el daño crónico en Lupus Eritematoso Sistémico: una asociación precoz y nociva. Estudio exploratorio.
}

Alvaro Danza

ORCID: 0000-0001-9070-2230 Internista. Farmacólogo. Profesor Agregado Clínica Médica. Hospital

Pasteur.

Jorge Narváez ORCID: 0000-0002-3655-2734 Internista.

Diego Graña ORCID: 0000-0001-8979-4692 Internista. Profesor Adjunto Clínica Médica. Hospital Pasteur.

Leticia Pérez ORCID: 0000-0002-0177-2307 Residente medicina interna. Hospital Pasteur.

Alexander Viera ORCID: 0000-0002-3540-895X Residente medicina interna. Hospital Pasteur.

Augusto Baccelli ORCID: 0000-0001-5866-3120 Internista. Asistente Clínica Médica. Hospital Pasteur

Ignacio Borgia ORCID: 0000-0002-8562-0481 Internista. Ex Asistente Clínica Médica. Hospital Pasteur.

Federico Roca ORCID: 0000-0002-8666-4056 Internista. Profesor Adjunto Clínica Médica. Hospital Pasteur.
Glucocorticoids related damage in Systemic Lupus Erythematosus: an early and harmful association. An exploratory analysis.

Relação entre o uso de glicocorticóides e dano crônico no Lupus Eritematoso Sistêmico: uma associação precoce e prejudicial. Estudo exploratório.

Resumen: Introducción. La actividad inflamatoria persistente el Lupus Eritematoso Sistémico provoca daño permanente. El daño permanente puede ser atribuido a la enfermedad y/o al tratamiento, en particular los glucocorticoides. El objetivo de este trabajo fue conocer la relación entre el daño crónico y la exposición a glucocorticoides. Material y métodos. Se realizó un muestreo no probabilístico de pacientes con lupus eritematoso sistémico. Se analizaron variables demográficas, niveles de actividad, dosis prednisona iniciales y acumuladas y niveles de daño medidos por "SLICC Damage Index" en diferentes periodos de la enfermedad. El daño fue clasificado en "relacionado" y "no relacionado" con el uso de glucocorticoides. Resultados. Se analizaron 30 pacientes, todos de sexo femenino. La media de seguimiento fueron 155 (DE 127) meses. Al final del seguimiento 13/30 (43,3\%) pacientes presentaron daño orgánico. Los pacientes que presentaron daño orgánico "relacionado" con glucocorticoides al final del seguimiento presentaron dosis de inicio de prednisona significativamente mayores que los que no presentaron daño 53,3 (DE 10,3) mg/día vs. 28, 3 (DE 24) mg/día, p<0,05. Dosis de inicio mayores a $30 \mathrm{mg} /$ día se relacionaron con el daño al final del seguimiento, independientemente de los niveles de actividad considerados, OR 2,05 (IC 95\% 1,5-4,0). Las dosis acumuladas de prednisona mayores a $3000 \mathrm{mg}$ en el primer año se asociaron con daño relacionado con glucocorticoides al final del seguimiento $(p<0,05)$. Conclusiones. Existe una acumulación de daño a lo largo del tiempo relacionado con los glucocorticoides. Esta relación es precoz, esto es, las dosis de inicio pueden relacionarse con la acumulación de daño a largo plazo, particularmente considerando los dominios relacionados con glucocorticoides, independientemente del nivel de actividad considerado.

Palabras clave: Glucocorticoides, Lupus eritematoso sistémico, Efectos colaterales y reacciones adversas relacionadas con medicamentos

Abstract: Background. Persistent activity causes irreversible organ damage in Systemic Lupus Erythematosus (SLE). Permanent damage can be attributed to the disease and to the treatment, particularly glucocorticoids. We aimed to know the relationship between the presence of organ damage and the exposure to glucocorticoids (GCC). Methodology. A non-probabilistic retrospective study of patients with SLE was performed. Demographic variables, activity levels, initial, accumulated prednisone dose and damage measured by "SLICC Damage Index (SDI)" at various stages from the diagnosis of the disease, were analyzed. Damage was classified in related and not related to GCC. Results. Thirty patients were analyzed, all women. The mean follow-up was 155 (SD: 127) months. At the end of follow-up 13/30 (43.3\%) patients 
presented organ damage. Patients who had GCC-related damage at the end of follow-up had a significantly higher mean starting dose of prednisone, 53.3 (SD: 10.3) mg/d vs. 28.3 (SD: 24) $\mathrm{mg} / \mathrm{d}, \mathrm{p}<0.05$. The effect on damage was observed with prednisone starting dose greater than $30 \mathrm{mg} / \mathrm{d}$, regardless of the level of activity at the onset of the disease, OR 2.05 (Cl 95\% 1.5 - 4.0). Cumulative doses of prednisone at one year greater than $3000 \mathrm{mg}$, were related to GCC-related damage at the end of the follow-up $(p<0.05)$. Conclusions. There is an accrual of damage over time associated to glucocorticoids exposure. It is highlighted that the relationship is early, that is, the starting dose will probably signify the accumulation of damage, especially in glucocorticoidrelated domains, regardless of activity levels.

Key words: Systemic lupus erythematosus, Glucocorticoids, Treatment safety, Chronic damage

Resumo: Introdução. A atividade inflamatória persistente do Lúpus Eritematoso Sistêmico (LES) causa danos permanentes. Danos permanentes podem ser atribuídos a doenças e / ou tratamento, particularmente glicocorticóides. O objetivo deste trabalho foi conhecer a relação entre dano crônico e exposição a glicocorticóides. Material e métodos. Foi realizada uma amostragem não probabilística de pacientes com LES. Variáveis demográficas, níveis de atividade, doses iniciais e acumuladas de prednisona e níveis de dano medidos pelo "SLICC Damage Index (SDI)" foram analisados em diferentes períodos da doença. O dano foi classificado como "relacionado" e "não relacionado" ao uso de glicocorticóides.Resultados. 30 pacientes, todos do sexo feminino, foram analisados. O seguimento médio foi de 155 (DP 127) meses. Ao final do seguimento 13/30 (43,3\%) pacientes apresentavam lesão orgânica. Os pacientes que apresentaram lesão orgânica "relacionada" aos glicocorticoides ao final do seguimento tiveram doses iniciais de prednisona significativamente maiores do que aqueles que não apresentaram lesão: 53,3 (DP 10,3) mg / dia vs. 28,3 (SD 24) mg / dia, $p<0,05$. Doses iniciais maiores que 30 $\mathrm{mg} /$ dia foram relacionadas a danos ao final do seguimento, independentemente dos níveis de atividade considerados, OR 2,05 (IC 95\% 1,5 - 4,0). Doses cumulativas de prednisona maiores que $3000 \mathrm{mg}$ no primeiro ano foram associadas a danos relacionados aos glicocorticoides no final do acompanhamento $(p<0,05)$. Conclusões. Há um acúmulo de danos ao longo do tempo relacionados aos glicocorticóides. Essa relação é precoce, ou seja, as doses iniciais podem estar relacionadas ao acúmulo de danos em longo prazo, principalmente considerando os domínios relacionados aos glicocorticóides, independentemente do nível de atividade considerado.

Palavras-chave: Glicocorticóides, Lúpus eritematoso sistêmico, Efeitos colaterais e reações adversas relacionadas a medicamentos

Recibido: 28/10/2020 Aceptado: 19/02/202

Clínica Médica 2. Hospital Pasteur. Facultad de Medicina. Universidad de la República. Administración Servicios de Salud del Estado (ASSE). Montevideo, Uruguay. 


\section{Introducción}

El Lupus Eritematoso Sistémico (LES) es una enfermedad autoinmune sistémica de presentación clínica heterogénea ${ }^{(1)}$. Si bien se puede presentar a cualquier edad, frecuentemente se observa mujeres en edad reproductiva ${ }^{(2)}$. En Sudamérica, la tasa estimada de incidencia es alrededor de 1 a 25 casos por cada 100.000 habitantes, con tasas de prevalencia que varían entre 20-70 casos por 100.000 habitantes, documentándose mayor prevalencia y severidad en poblaciones afrocaribeñas ${ }^{(3,4)}$.

El objetivo terapéutico en el LES consiste en disminuir de forma precoz, efectiva y sostenida la actividad de la enfermedad. Es bien conocido que la actividad persistente provoca daño orgánico irreversible y que, a mayor actividad mayor daño. A su vez, el daño acumulado provoca diversos grados de morbilidad y aumenta la mortalidad en el LES ${ }^{(5,6)}$.

El daño en el LES se define como la pérdida de funcionalidad de los órganos o sistemas afectados, tiene un carácter irreversible, y puede ser resultado directo de la actividad de la enfermedad, de los medicamentos empleados para el tratamiento, o bien asociada a enfermedades preexistentes ${ }^{(7-9)}$.

Se estima que aproximadamente dos tercios de los pacientes afectados presentará algún nivel de daño transcurridos 7 años desde el diagnóstico de la enfermedad (7-10). Se han diseñado instrumentos para la medición del daño en esta enfermedad, por su sigla en inglés "Systemic Lupus International Collaborating Clinics/American College of Rheumatology Damage Index", en adelante "SDI", que establecen categorías y órganos afectados. El daño debe estar confirmado por un método diagnóstico objetivo y estar presente por al menos 6 meses para definir su presencia ${ }^{(10)}$.

De igual forma, se han diseñado instrumentos para medir los niveles de actividad de la enfermedad. Uno de ellos, ampliamente empleado, es el SLEDAI-2K, por su sigla en inglés "Systemic Lupus Erythematosus Disease Activity Index-2000" (10).

Los pilares del tratamiento del LES se basan en el uso de Hidroxicloroquina, inmunosupresores, glucocorticoides y agentes biológicos ${ }^{(5)}$. Hidroxicloroquina es una antimalárico de síntesis cuya eficacia en el tratamiento del LES es hoy indiscutida. Diversos grupos han confirmado que disminuye el riesgo de trombosis y la mortalidad, tiene un efecto protector sobre las infecciones y es un ahorrador de corticoides, entre otras características ${ }^{(11)}$. Dentro de los inmunosupresores destacan Metotrexato para las manifestaciones cutáneas, articulares y serosas. Azatioprina, fundamentalmente para manifestaciones renales, del Sistema Nervioso y hematológicas ${ }^{(12)}$. En los últimos tiempos ha irrumpido Micofenolato de Mofetilo como un agente inmunosupresor eficaz para el tratamiento de inducción y mantenimiento de la Nefritis Lúpica $(12,13)$. Sin embargo, su uso se ha ampliado extensamente para manifestaciones "fuera de etiqueta", utilizándoselo como un inmunosupresor de primera línea en manifestaciones graves y de segunda línea cuando fallan alguno de los anteriormente mencionados (12 - 14).

El arsenal de terapias biológicas en el LES es de menor amplitud que en otras enfermedades inflamatorias y autoinmunes, pero igualmente promisorio. En efecto, existen dos moléculas ampliamente empleadas, Rituximab y Belimumab. El análisis de su utilidad excede ampliamente el alcance de este trabajo, pero vale la pena recordar que el primero de ellos suele ser empleado en casos refractarios o corticodependientes de manifestaciones severas (sistema nervioso central, nefritis, vasculitis, hematológicas) mientras que el segundo en casos refractarios o corticodependientes a tratamientos convencionales, fundamentalmente de la esfera articular, cutánea y serosa $(15,16)$.

El uso de glucocorticoides (GCC) es ampliamente difundido en el tratamiento del LES, en distintas etapas de la enfermedad, ya sea en remisión o, particularmente, en períodos de actividad. Los pacientes suelen estar expuestos por períodos variables, habitualmente prolongados. En períodos de actividad se recurre a su efecto inmunosupresor y antiinflamatorio rápido y efectivo; mientras que en períodos de remisión se propone mantener estos efectos. Su elevada efectividad antiinflamatoria e inmunosupresora contrasta con su perfil de seguridad, caracterizado por una gran amplitud de efectos adversos. En efecto, buena parte de estos efectos adversos, acumulados en el tiempo, constituyen y contribuyen al daño orgánico crónico orgánico e irreversible $(17,18)$.

Es bien conocido que los efectos adversos de los GC son múltiples y afectan, de manera variable, a casi todos los órganos y tejidos del organismo. Está bien establecido que existe una relación lineal entre la dosis y la duración del tratamiento y la aparición de ciertos efectos adversos $(19,20)$. Además, no existe evidencia que respalde la eficacia de dosis altas. Su uso, 
casi constante, ha contrastado con la falta de protocolos claros sobre dosis máxima, pautas de descenso y suspensión de estos. (19-22)

En las últimas décadas se ha observado de forma sistemática que dosis menores a las "habituales o convencionales" de prednisona, de $1 \mathrm{mg} / \mathrm{kg} /$ día, son igualmente efectivas e indiscutiblemente más seguras. El desafío, entonces, consiste en identificar la mejor relación beneficio/riesgo de los tratamientos empleados para provocar el menor daño a largo plazo (22).

Este "viejo paradigma" del uso de GC, particularmente del uso de prednisona a $1 \mathrm{mg} / \mathrm{kg} /$ día ha marcado a generaciones de médicos. Nada tan arraigado a la práctica profesional como carente de evidencia. Nuestro grupo ha trabajado firmemente en los últimos años sobre este tema. En efecto, hemos confirmado que dosis menores son igualmente efectivas, determinando remisiones precoces y sostenidas en el tiempo y determinando un menor perfil de efectos adversos (20-22).

Dadas las diversas manifestaciones de esta enfermedad y los múltiples posibles riesgos asociados al uso de glucocorticoides, nos propusimos conocer la relación entre la presencia de daño orgánico y su asociación con la exposición a los GC.

\section{Material y Métodos}

Se realizó un estudio retrospectivo de pacientes con LES, según criterios clasificatorios del ACR $1997^{(23)}$ de un único centro, asistidos en el Hospital Pasteur de la ciudad de Montevideo. El Hospital Pasteur es un hospital de tercer nivel asistencial polivalente, de adultos, que cuenta con 220 camas totales, entre cuidados moderados y especializados. Dispone de un policlínico de referencia para ciertas enfermedades que asiste una población total de 100.000 usuarios. Se identificaron todos los pacientes en seguimiento por LES en dicho centro asistencial.

Se analizaron variables demográficas (sexo y edad), tabaquismo, niveles de actividad medido por score SLEDAI-2K, definido en diversos momentos desde el diagnóstico de la enfermedad: T0, al inicio de la enfermedad; T1, al mes; T2, a los 6 meses; T3 a los 12 meses y T4 a los 5 años o al final del seguimiento; dosis de prednisona con que comenzó el tratamiento, acumulada en T1, T2, T3, T4 (con igual criterio cronológico que en el SLEDAI-2K); daño en T2, T3 y T4 medido por estimador de daño „SLICC Damage Index (SDI)“ $(7,23)$.

Se excluyeron los casos en los cuales el daño no podía ser estimado, la dosis de glucocorticoides no podía ser verificada, los casos con nefritis lúpica, diabetes, con creatinina mayor oigual a $1,3 \mathrm{mg} / \mathrm{dl}$ y los que presentaran daño permanente previo a ser controlados en nuestra Unidad.

EI SLEDAI-2K, es un instrumento que permite la revision por sistemas (nervioso central y periférico, cardiovascular, nefrológico, esquelético entre otros), en el interrogatorio, examen físico y analítica del paciente. A cada item se le otorga un puntaje, que finalmente se suma y arroja un puntaje final ${ }^{(24)}$. Dicha puntuación va de 0 a 105 (valor teórico); no obstante, la manifestación de uno o dos signos o síntomas mayores, se catalogará como actividad severa, aunque esta puntuación sea baja y los síntomas restantes se ubiquen en categorías más bajas, ya que prima la importancia de los signos mayores sobre la puntuación y demás signos menores presentes. Para este trabajo se consideró actividad leve: $\leq$ a 4 puntos, moderada $>4$ puntos pero $<8$, severa $\geq$ a 8 puntos. La remisión se define como un puntaje igual a 0 y se considera sostenida si persiste más de 3 meses.

El SDI por su sigla en inglés (Índice de daño) establece el grado de daño tisular desde el inicio de la enfermedad, el cual puede ser resultado de enfermedad previa, intercurrente o tratamiento, y evalúa por separado 12 diferentes sistemas, siendo necesario que los signos y síntomas se manifiesten al menos seis meses previos a la realización de dicha prueba ${ }^{(7)}$.

Para simplificar el análisis se dicotomizó el daño permanente en "relacionado con GCC" (cataratas, osteonecrosis, fractura osteoporótica, diabetes mellitus y miopatía) y "no relacionado con GCC" (neuropsiquiátrico, renal, pulmonar, ocular no catarata, cardiovascular central o periférico, gastrointestinal, musculoesquelético, cutáneo, fallo gonadal prematuro, malignidad), según lo recomendado en la literatura ${ }^{(25)}$.

Las variables cuantitativas se presentan con medidas de tendencia central y dispersión; media y desvío estándar y mediana y rango, según su distribución. La normalidad se probó mediante test de Hipótesis (Shapiro-Wilk) y gráficos. Las variables cualitativas se presentan mediante frecuencias absoluta y relativa. Las comparaciones entre grupos se realizarán mediante Chi2 o Test de Fisher para los datos cualitativos y mediante Test de t de Student o de Wilcoxon, según la distribución, para los datos cuantitativos. Para conocer la influencia de los 
niveles de actividad en las dosis de prednisona y su relación con el daño orgánico a largo plazo se realizó un análisis de regresión logística binaria. Se tomó el daño como variable dependiente, dicotomizada en "presente o ausente". Se consideró un nivel de significación estadística menor a 0.05 . Los datos se procesaron en el programa informático SPSS.

El trabajo se desarrolló de acuerdo con la Declaración de Helsinki de la Asociación Médica Mundial. Se obtuvo autorización del Comité de Ética Institucional. Los datos fueron tratados con total confidencialidad, para lo cual se anonimizó la información de los participantes.

\section{Resultados}

Se analizaron 30 casos, todos de sexo femenino, media de edad 46,3 (DE: 12,9) años, 7/30 fueron fumadoras. La media de seguimiento fue 155 (DE: 127) meses.

Con relación al perfil de autoanticuerpos: 8/27 presentaron anti-Smith; 14/27 anti-Ro/La; 6/28 anti-RNP; anticuerpos antifosfolípidos estuvieron presentes en 4/30 casos (Tabla 1).

\begin{tabular}{|l|l|}
\hline & Número de casos $=\mathbf{3 0}$ \\
\hline Sexo femenino & 30 \\
\hline Edad (media y DE), en años & $46,3(12,9)$ \\
\hline Anti-Smith & $8 / 27(29,6 \%)$ \\
\hline Anti-Ro/La & $14 / 27(51,8 \%)$ \\
\hline AFL ${ }^{*}$ & $4 / 30(13,3 \%)$ \\
\hline Reciben PDN al inicio del seguimiento & $22 / 30(73,3 \%)$ \\
\hline Reciben PDN > 30 mg/d al inicio del seguimiento & $16 / 30(53,3 \%)$ \\
\hline SLEDAl-2K en T0 (media, DE) & $8,86(7)$ \\
\hline
\end{tabular}

Abreviaturas. $\mathrm{DE}=$ desvío estándar; $\mathrm{AFL}=$ anticuerpos antifosfolípidos; $\mathrm{PDN}=$ prednisona.

Los anticuerpos anti-DNA fueron positivos en 14/27 pacientes en T0; 7/27 en T1; 6/27 en T2 y 4/27 en T3 y $3 / 27$ en T4, p< 0,05 entre T0 y T4. La media de SLEDAI en T0 fue de 8,86 (DE: 7), T1: 5,93 (DE: 5), T2: 4,37 (DE: 5), T3: 3,53 (DE: 4,6), T4 2,33 (DE: 3,4); $p<0,05$ entre los periodos (Gráfico 1).

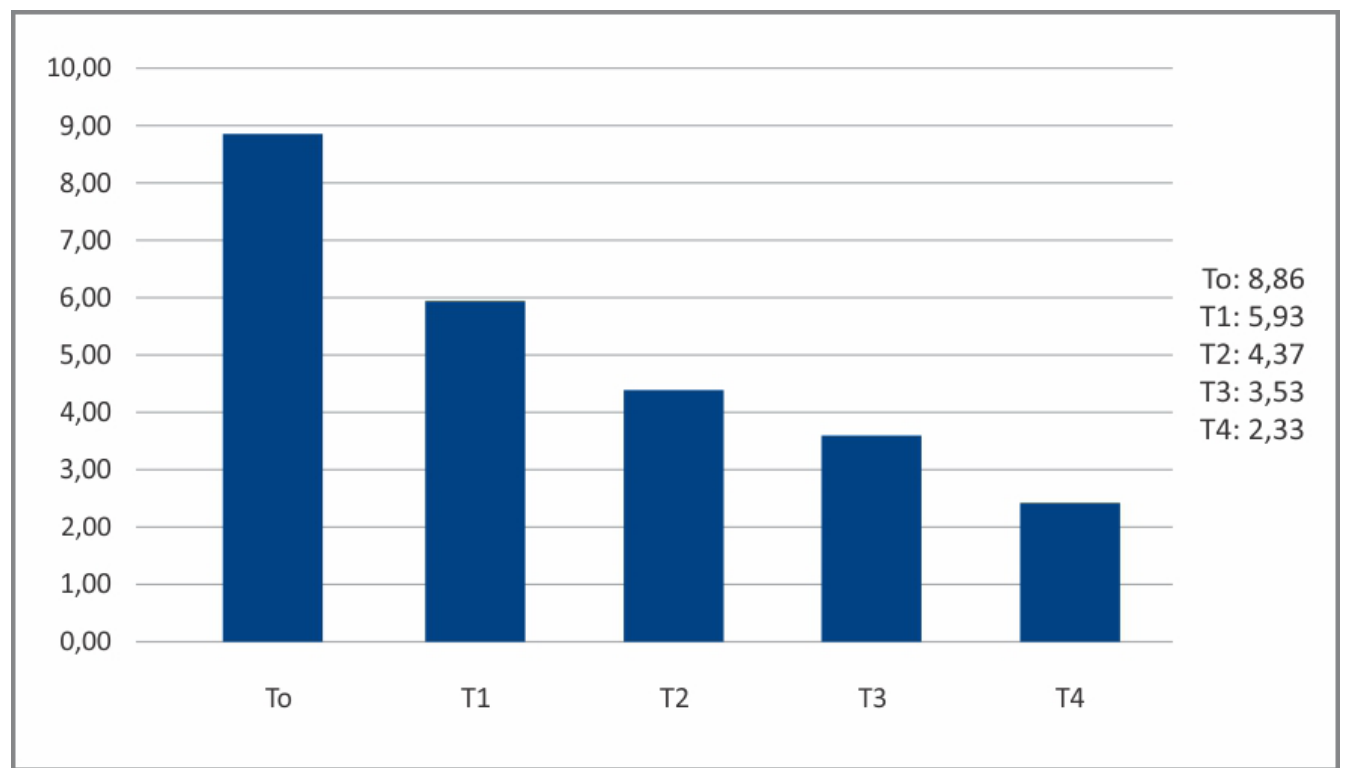

T0: al inicio del tratamiento; T1: al mes de tratamiento; T2: a los 6 meses de tratamiento; T3: a los 12 meses de tratamiento y T4 a los 5 años o final del seguimiento. $\mathrm{p}<0.05$ entre los períodos.

Veintidós pacientes de 30 (73,3\%) fueron tratados con prednisona al inicio de la enfermedad. La media de dosis máxima de prednisona al inicio del tratamiento fueron 33,9 (DE 23,8) mg/día; la media de dosis acumulada en T1 (al mes) fue 1085 (DE 1011) mg; en T2 (a los 6 meses) fue de 4125 (DE 3400) mg; a los 12 meses (T3) fue 6314 (DE 5868,4) mg y en T4 (5 años) fue de 7270 (DE 7568,21) mg, rango intercuartílico: 315 - 11235 mg. 
Las medianas (intervalo intercuartílico) de SDI observadas en T2 fue 0 (0-1), en T3: 1 (1-1,25, T4: 2 (0-2). Al final del seguimiento 13/30 (43.3\%) pacientes presentaron algún nivel de daño orgánico.

Los pacientes que presentaron daño en los dominios relacionados con GCC en T4 tuvieron una media de dosis inicial significativamente superior, 53,3 (DE: 10,3) mg/d vs. 28,3 (DE: 24) $\mathrm{mg} / \mathrm{d}$, de los que no presentaron daño, $\mathrm{p}<0,05$ (Gráfico 2).

Gráfico 2: Media e intervalo de confianza $95 \%$ de dosis de prednisona al inicio del tratamiento en pacientes que presentan daño permanente por glucocorticoides al final del seguimiento y los que no lo presentan $(p<0,05)$.

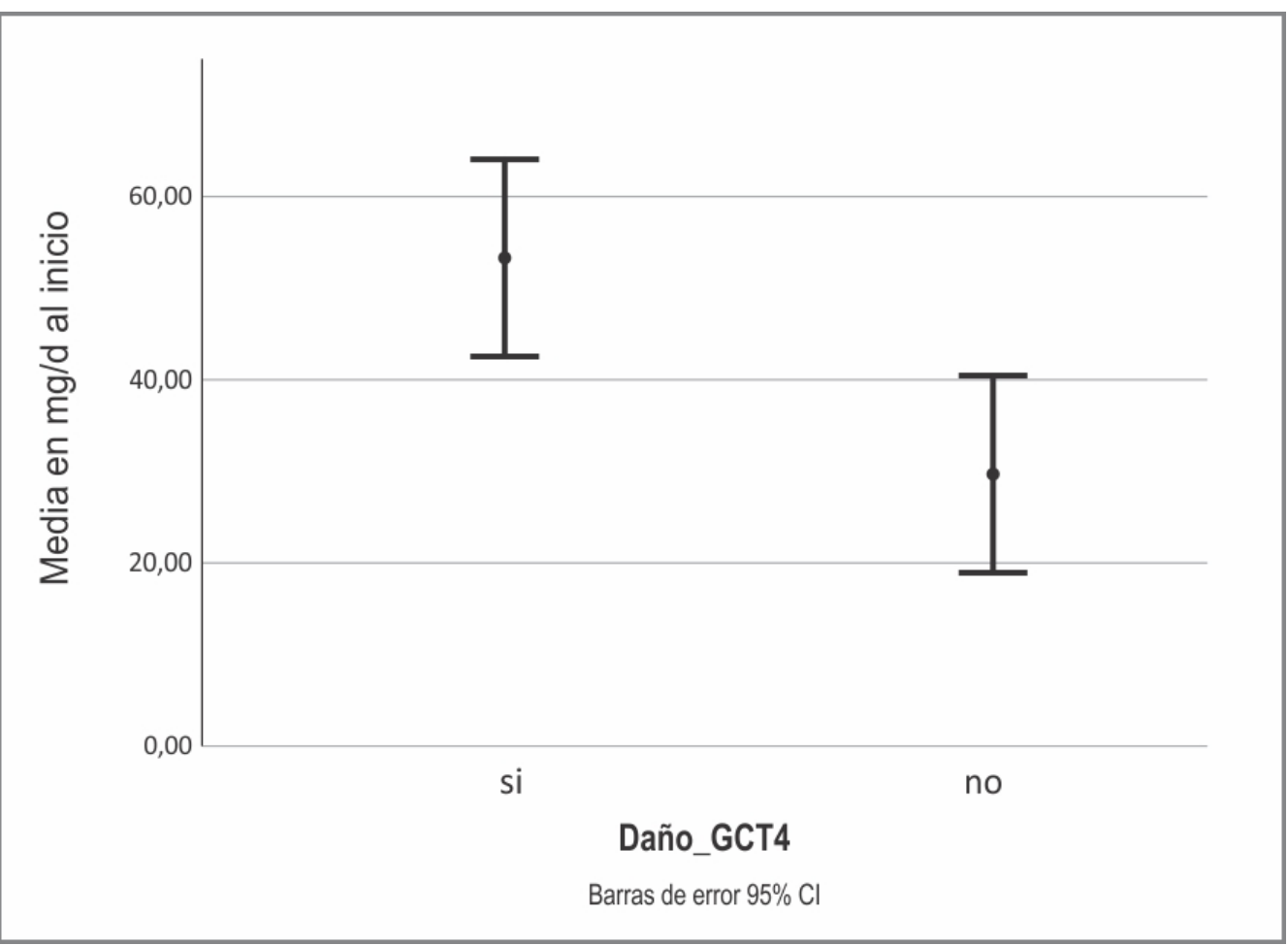

La media de dosis acumulada de prednisona en T1 (1 mes de tratamiento) de los pacientes que presentaron daño relacionado con GCC en T4 fue 1493 (289) mg respecto a los que no presentaron daño, que fue 963 (1120) $\mathrm{mg}, \mathrm{p}<0,05$. La media de dosis acumulada (DE) en T2 (6 meses de tratamiento) entre los que presentaron daño relacionado con GCC en T4 (5 años) fue 6930 (3639) mg, mientras que los que no presentaron daño relacionado con GCC en igual plazo fue de 3190 (2835) mg, p < 0,05. La medida de dosis acumulada (DE) en T3 (1 año) entre los que presentaron daño al final del seguimiento (T4) fue $8362,3(5941,2) \mathrm{mg}$, mientras que en los que no presentaron daño fue $4198,2(4913,8)$ mg considerado el mismo período de seguimiento $(p=$ $0,08)$.

No hubo diferencias estadísticamente significativas en la media de SLEDAI en T4 en los pacientes que presentan daño relacionado con GCC: 1,33 (DE: 3,26) y los que no presentaron daño: 2,73 (DE: 3,55), p>0,05.

En la tabla 2 se presenta el plan de tratamiento con GC en el grupo de pacientes con LES.

\begin{tabular}{|c|c|c|}
\hline Primer mes & $\begin{array}{l}\text { PDN } 30 \text { mg/d (15 días) } \\
\text { PDN } 20 \text { mg/d (15 días) }\end{array}$ & \multirow{4}{*}{$\begin{array}{l}\text { Iniciar precozmente siempre: } \\
\text { - Hidroxicloroquina (en } \\
\text { ausencia de contraindicaciones, } \\
\text { excepcional) } \\
\text {-Inmunosupresor de } \\
\text { mantenimiento (AZA, MTX, CFM } \\
\text { o MMF según el caso) } \\
\text {-Pulsos de MTP si brote (no } \\
\text { mayores a } 500 \text { mg/d por } 3 \text { días) }\end{array}$} \\
\hline Segundo mes & $\begin{array}{l}\text { PDN } 15 \text { mg/d (15 días) } \\
\text { PDN } 10 \text { mg/d (15 días) }\end{array}$ & \\
\hline Tercer mes & PDN 7,5 mg/d (1 mes) & \\
\hline Cuarto mes en adelante & $\begin{array}{l}\text { PDN } 5 \text { mg/d (mínimo } 1 \text { mes) } \\
\text { Intentar suspender } \\
\text { Recordar llegar a } 5 \text { mg/d en } 12 \text { - } \\
14 \text { semanas }\end{array}$ & \\
\hline
\end{tabular}


En el análisis de regresión logística binaria, tomando como variable dependiente el daño inducido por corticoides en los dominios atribuidos a éstos (daño presente o ausente) al final del seguimiento (T4), el efecto sobre el daño se observó con dosis de inicio de prednisona mayores a $30 \mathrm{mg} / \mathrm{d}$, independientemente del nivel de actividad medido por SLEDAl-2K en el inicio de la enfermedad (T0), $p>0.05$, OR 2,05 (IC 95\% 1,5-4,0). De igual modo, las dosis acumuladas de prednisona al año (T3) mayores a $3000 \mathrm{mg}$, se mostraron relacionadas con el daño vinculado al uso de corticoides en los dominios atribuidos a éstos al final del seguimiento (T4), independientemente del nivel de actividad al mes (SLEDAI-2K, T1), p > 0.05. Sin embargo, el análisis de regresión evidenció que el daño en los dominios no atribuidos a los corticoides al final del seguimiento estuvo influenciado por los niveles de actividad iniciales $(p<0.05)$ independientemente de las dosis de corticoides empleadas al inicio y acumuladas al año.

En relación con el uso de Hidroxicloroquina, se constató que 22/30 (73,3\%) recibieron Hidroxicloroquina. En cambio, 2/30 (6,7\%) recibieron Metotrexato, 13/30 Azatioprina (43,3\%), $8 / 30$ Micofenolato de Mofetilo $(26,7 \%)$ y $5 / 30$ (16,7\%) recibieron Ciclofosfamida. Es de destacar que el dato refiere a si alguna vez recibieron alguno de estos inmunosupresores.

\section{Discusión}

Nuestro trabajo muestra una asociación entre el uso de glucocorticoides y el daño permanente en una cohorte de pacientes con Lupus Eritematoso Sistémico. La relación se establece particularmente entre dosis elevadas al inicio y el daño permanente en los dominios relacionados con glucocorticoides del SDI al final del seguimiento.

Es de destacar que, en la captación, y por lo tanto al inicio del tratamiento (T0) los niveles de actividad fueron elevados, lo que condicionó probablemente el uso de dosis altas de glucocorticoides, en particular prednisona. Su eficacia en disminuir la actividad es indiscutible. En efecto, se observa una disminución clara de los niveles de actividad en los distintos cortes temporales (un mes, seis meses, al año y a los cinco años), con diferencia estadística entre sí.

Estas observaciones son consistentes con los objetivos del tratamiento del LES, que implican, entre otros aspectos, disminuir los niveles de actividad de la enfermedad, de forma precoz y sostenida en el tiempo ${ }^{(5)}$. Se dispone de una amplia gama de terapéutica para ello, pero los glucocorticoides siguen concitando atención por su rápido y eficaz efecto antiinflamatorio.

Nuestras observaciones están en línea con trabajos pioneros sobre este tema. En el año 2000, el grupo de la cohorte del John Hopkins, que lidera Michele Petri, con más de 1000 pacientes durante más de 30 años, comprobó que las dosis acumuladas de prednisona se relacionaban con diferencias estadísticamente significativas con osteonecrosis, enfermedad coronaria y cataratas. A su vez, cada 2 meses de exposición a dosis elevadas (mayores a 60 mg/día) aumentó un 20 \% el riesgo de necrosis avascular de la cabeza femoral y ataque cerebrovascular. Estos efectos no se comprobaron para el uso de pulsos de metilprednisolona al inicio del tratamiento $(18,26)$.

Estas observaciones fueron corroboradas en estudios posteriores del grupo del Hospital de Cruces (Bilbao). Ruiz-Irastorza et al. observaron un 38\% de daño acumulado a 5 años, comparable a nuestras observaciones. Asimismo, comprobaron que los pacientes que recibían dosis medias o altas de glucocorticoides, mayores a tan sólo $7,5 \mathrm{mg} / \mathrm{día}$ al final del cuarto año de seguimiento, presentaron un OR de 5,39 (IC 95\% 1,59 -18,27) de presentar daño permanente, en dominios relacionados y no relacionados con glucocorticoides.

Llamativamente, y en línea con otras observaciones, los pulsos de metilprednisolona no se relacionaron con riesgo de daño permanente en ninguno de los dominios $(27,28)$.

En el mismo sentido, Apostolopoulos et al., en una cohorte de 162 pacientes, observaron datos similares a los nuestros, encontrando que $75 \%$ de los pacientes habían recibido prednisolona y que más de la mitad presentaban algún nivel de daño en 7 años, con valores comparables a los nuestros. Los autores encontraron una asociación firme entre la exposición a glucocorticoides y la probabilidad de desarrollar daño, observando que a mayores dosis mayores daño, independientemente de si se consideraran dominios relacionados o no con glucocorticoides. Esta asociación se mostró independientemente del nivel de actividad de la enfermedad, algo similar a lo que ocurrió en nuestro trabajo ${ }^{(29)}$.

Nuestros datos confirman una relación entre la dosis de glucocorticoides y el daño inducido por éstos, de forma dosis-dependiente. En efecto, dosis de inicio mayores a $30 \mathrm{mg} / \mathrm{día}$ se asociaron con el doble de chance de daño en los dominios relacionados con glucocorticoides al final del seguimiento. Esta observación no se confirmó para los dominios no relacionados con glucocorticoides. En la regresión logística, esta asociación persistió, independientemente 
de los niveles de actividad considerados. De igual modo, dosis acumuladas al año por encima de $3000 \mathrm{mg}$ se asociaron con daño al final del seguimiento en los dominios relacionados con glucocorticoides, no así en los no relacionados.

Curiosamente, en nuestra casuística, ningún paciente recibió pulsos de metilprednisolona, algo otrora planteado sólo para casos graves a muy graves. De igual modo, si bien es conocido que el uso de hidroxicloroquina es la piedra angular del tratamiento del LES con una eficacia indiscutible y un perfil de seguridad muy favorable, llama la atención que casi una cuarta parte no la recibieran ${ }^{(30)}$. La mayoría de los pacientes $(28 / 30)$ recibieron algún inmunosupresor para el tratamiento. El diseño del trabajo no permite identificar si la introducción fue precoz, dentro del primer mes de diagnóstico, o tardía. En el primer caso, permitirían ahorrar corticoides y evitar la acumulación de dosis elevadas al final del primer año.

Ninguna de estas dosis elegidas es casual. La primera, $30 \mathrm{mg} /$ día de prednisona, es la dosis que, nuestro grupo, ha venido postulando como "dosis máxima" de prednisona vía oral, en contraposición a los clásicos esquemas de $1 \mathrm{mg} / \mathrm{kg} / \mathrm{día}$. La segunda, porque nuestro esquema terapéutico postula no acumular dosis mayores a 3 gramos en un año, en particular durante el primer año de seguimiento (Tabla 2).

Para lograr esto, es fundamental introducir precozmente inmunosupresores y asociar pulsos de metilprednisolona para evitar emplear dosis elevadas (mayores a $30 \mathrm{mg} / \mathrm{día}$ ) de prednisona. En efecto, diversos trabajos concluyen que el uso de metilprednisolona permite emplear dosis más bajas de prednisona "de salida" y no se han relacionado con daño orgánico permanente $(27-29)$.

Estas consideraciones están basadas, no sólo en observaciones clínicas bien documentadas, sino que tienen sólidos fundamentos farmacológicos. Los glucocorticoides actúan por dos vías: genómica y no genómica $(31,32)$. La primera es la vía "clásica", por la cual la molécula del glucocorticoide se une a un receptor citosólico que es trasportado al núcleo celular y allí regula, en más o en menos, la transcripción genética de moléculas involucradas en el proceso inflamatorio y en los efectos adversos. Esta vía está expresada en todas las células del organismo y se ha postulado que tiene su techo antiinflamatorio a dosis de prednisona o equivalente de 30 a $40 \mathrm{mg}$. Luego de estas dosis la capacidad de modular en menos la transcripción génica de moléculas vinculadas al proceso inflamatorio es prácticamente nula, al tiempo que su capacidad de modular moléculas relacionadas con los efectos adversos, está activa y es dosis-dependiente. La vía no genómica, más novedosa, estaría especialmente expresada en las células inflamatorias y consiste en la modulación de un receptor de membrana y de vías intracelulares no relacionadas con el receptor citosólico de los glucocorticoides. Esta vía se activa con dosis de prednisona o equivalente mayores a $100 \mathrm{mg}$ y es especialmente sensible a dos moléculas, metilprednisolona y dexametasona. Esta es la razón por la cual nuestro grupo promueve el uso de estas moléculas en los brotes, en lugar de dosis de prednisona mayores a 30-40 mg, que no logran reclutar la vía no genómica y a la cual la vía genómica está reclutada de forma casi completa (31-33).

Nuestro trabajo tiene debilidades por su carácter retrospectivo, el muestreo no probabilístico considerado y el reducido número de casos. Probablemente, esta es la razón por la cual no pudimos comprobar el efecto protector del uso de Hidroxicloroquina sobre el daño relacionado al uso de glucocorticoides, observado por otros autores. Sin embargo, es la primera vez que en nuestro medio se explora la relación entre el daño orgánico en lupus y la exposición a los glucocorticoides. En efecto, se observa una relación precoz con el daño a largo plazo.

\section{Conclusiones}

Si bien los niveles de daño en nuestra cohorte son bajos, se observa un aumento con el tiempo y una relación clara con la exposición al uso de GCC. Se destaca que la relación es temprana, es decir, la dosis de inicio probablemente signará la acumulación de daño, especialmente en los dominios relacionados con glucocorticoides, independientemente de los niveles de actividad. Existe evidencia creciente que dosis más bajas pueden ser igualmente efectivas y más seguras. Deben continuarse estas exploraciones para establecer conclusiones más robustas.

\section{Conflictos de Interés}

No hay conflictos de interés para declarar con relación a este estudio. 


\section{Agradecimientos}

A la Dra. Andrea Vargas, Profesora Adjunta de la Clínica de Reumatología de la Facultad de Medicina de la Universidad de la República y Reumatóloga del Hospital Pasteur, por facilitar acceso a la base de datos empelada en este trabajo.

\section{Bibliografía}

1- D’ Cruz D, Khamashta M, Hughes GRV. Systemic lupus erythematosus. Lancet. 2007; 369: 587- 96.

2- Jiménez S, Cervera R, Font J, Ingelmo M. The epidemiology of systemic lupus erythematosus. Clin Rev Allergy Immunol. 2003;25(1):3-12.

3- Pons-Estel BA, Catoggio LJ, Cardiel MH, Soriano ER, Gentiletti S, Villa AR, et al. The GLADEL multinational Latin American prospective inception cohort of 1,214 patients with systemic lupus erythematosus: ethnic and disease heterogeneity among "Hispanics". Medicine (Baltimore). 2004;83(1):1-17.

4- Pons-Estel GJ, Alarcón GS, Scofield L, Reinlib L, Cooper GS. Understanding the epidemiology and progression of systemic lupus erythematosus. Semin Arthritis Rheum. 2010; 39:257-68.

5- Fanouriakis A, Kostopoulou M, Alunno A, Aringer M, Bajema I, Boletis JN, et al. 2019 update of the EULAR recommendations for the management of systemic lupus erythematosus. Ann Rheum Dis. 2019;78(6):736-45.

6- Ruiz-Irastorza G, Danza A, Khamashta M. Tratamiento del lupus eritematoso sistémico: mitos, certezas y dudas. Med Clin (Barc). 2013;141(12):533-42.

7- Gladman D, Ginzler E, Goldsmith C, Fortin P, Liang M, Urowitz M, et al. The development and initial validation of the Systemic Lupus International Collaborating Clinics/American College of Rheumatology damage index for systemic lupus erythematosus. Arthritis Rheum. 1996;39:363-9.

8- Alba P, Gómez-Puerta JA, Goycochea-Robles MV, Amigo MC. Organ Damage and Quality of Life in Antiphospholipid Syndrome. Curr Rheumatol Rep. 2016;18(2):7.

9- Rivest C, Lew RA, Welsing PM, Sangha O, Wright EA, Roberts WN, et al. Association between clinical factors, socioeconomic status, and organ damage in recent onset systemic lupus erythematosus. $J$ Rheumatol. 2000; 27: 680-4.

10- Ruiz-Irastorza G, Danza A, Khamashta M. Glucocorticoids use and abuse in SLE. Rheumatology 2012; $51: 1145-53$

11- Ruiz-Irastorza G, Ramos-Casals M, Brito-Zeron P, Khamashta MA. Clinical efficacy and side effects of antimalarials in systemic lupus erythematosus: a systematic review. Ann Rheum Dis. 2010;69(1):20-28.

12- Pons-Estel BA, Bonfa E, Soriano ER, Cardiel MH, Izcovich A, Popoff F, et al. First Latin American clinical practice guidelines for the treatment of systemic lupus erythematosus: Latin American Group for the Study of Lupus (GLADEL, Grupo Latino Americano de Estudio del Lupus)-Pan-American League of Associations of Rheumatology (PANLAR). Ann Rheum Dis. 2018;77(11):1549-57.

13- Moroni G, Ponticelli C. Synthetic pharmacotherapy for lupus nephritis. Expert Opin Pharmacother. 2017;18(2):175-86.

14- Bertsias GK, Tektonidou M, Amoura Z, Aringer M, Bajema I, Berden JH, Boletis J, et al. Joint European League Against Rheumatism and European Renal Association-European Dialysis and Transplant Association (EULAR/ERA-EDTA) recommendations for the management of adult and paediatric lupus nephritis. Ann Rheum Dis. 2012;71(11):1771-82.

15- Mok CC. Current role of rituximab in systemic lupus erythematosus. Int J Rheum Dis. 2015;18(2):15463.

16- Thong B, Olsen NJ. Systemic lupus erythematosus diagnosis and management. Rheumatology (Oxford). 2017;56 (suppl1): i3-i13.

17- Ugarte A, Danza A, Ruiz-Irastorza G. Glucocorticoids and antimalarials in systemic lupus erythematosus: an update and future directions. Curr Opin Rheumatol. 2018;30(5):482-9.

18- Zonana-Nacach A, Barr SG, Magder LS, Petri M. Damage in systemic lupus erythematosus and its association with corticosteroids. Arthritis Rheum 2000; 43:1801-8.

19- Ruiz-Irastorza G, Olivares N, Ruiz-Arruza I, Martinez-Berriotxoa A, Egurbide MV, Aguirre C. Predictors of major infections in systemic lupus erythematosus. Arthritis Res Ther 2009; 11:R109. 
20- Danza A, Ruiz-Irastorza G. Infection risk in systemic lupus erythematosus patients: susceptibility factors and preventive strategies. Lupus. 2013; 22: 1286-94.

21- Ruiz-Irastorza G, Ugarte A, Ruiz-Arruza I, Khamashta M. Seventy years after Hench's Nobel prize: revisiting the use of glucocorticoids in systemic lupus erythematosus. Lupus. $2020 ; 29(10): 1155-1167$. doi: 10.1177/0961203320930099.

22- Ruiz-Irastorza G, Danza A, Perales I, Villar I, Garcia M, Delgado S, et al. Prednisone in lupus nephritis: how much is enough? Autoimmun Rev. 2014 Feb;13(2):206-14. doi: 10.1016/j.autrev.2013.10.013.

23- Hochberg MC. Updating the American College of Rheumatology revised criteria for the classification of systemic lupus erythematosus. Arthritis Rheum. 1997; 40:1725.

24- Gladman DD, Ibañez D, Urowitz MB. Systemic lupus erythematosus disease activity index 2000. J Rheumatol. 2002; 29(2):288-91.

25- Bañez D, Gladman D, Urowitz M. Summarizing disease features over time: II. Variability measures of SLEDAI-2K. J Rheumatol. 2007;34(2):336-40.

26- Al Sawah S, Zhang X, Zhu B, Magder LS, Foster SA, likuni N, Petri M. Effect of corticosteroid use by dose on the risk of developing organ damage over time in systemic lupus erythematosus-the Hopkins Lupus Cohort. Lupus Sci Med. 2015 Mar 11;2(1): e000066. doi: 10.1136/lupus-2014-000066.

27- Ruiz-Arruza I, Ugarte A, Cabezas-Rodriguez I, Medina JA, Moran MA, Ruiz-Irastorza G. Glucocorticoids and irreversible damage in patients with systemic lupus erythematosus. Rheumatology (Oxford). 2014;53(8):1470-6.

28- Ruiz-Arruza I, Barbosa C, Ugarte A, Ruiz-Irastorza G. Comparison of high versus low-medium prednisone doses for the treatment of systemic lupus erythematosus patients with high activity at diagnosis. Autoimmun Rev. 2015;14(10):875-9.

29- Apostolopoulos D, Kandane-Rathnayake R, Raghunath S, Hoi A, Nikpour M, Morand EF. Independent association of glucocorticoids with damage accrual in SLE. Lupus Sci Med. 2016;3(1):e000157.

30- Ruiz-Irastorza G, Khamashta MA. Hydroxychloroquine: the cornerstone of lupus therapy. Lupus. 2008;17(4):271-3.

31- Stahn C, Buttgereit F. Genomic and nongenomic effects of glucocorticoids. Nat Clin Pract Rheumatol. 2008;4(10):525-33.

32- Strehl C, Buttgereit F. Optimized glucocorticoid therapy: teaching old drugs new tricks. Mol Cell Endocrinol. 2013;380(1-2):32-40.

33- Apostolopoulos D, Morand EF. It hasn't gone away: the problem of glucocorticoid use in lupus remains. Rheumatology (Oxford). 2017;56(suppl_1): i114-i122.

\section{Aporte cada autor al trabajo}

Álvaro Danza: concepción y diseño del estudio, análisis, procesamiento e interpretación de los datos, redacción del borrador y revisión crítica del manuscrito.

Jorge Narváez: diseño del trabajo, recolección de datos, interpretación de los resultados, redacción del borrador.

Diego Graña: diseño del trabajo, recolección de datos, interpretación de los resultados, redacción del borrador.

Leticia Pérez: diseño del trabajo y recolección de datos.

Alexander Viera: diseño del trabajo y recolección de datos.

Augusto Baccelli: diseño del trabajo y recolección de datos.

Ignacio Borgia: diseño del trabajo y recolección de datos.

Federico Roca: concepción del estudio, interpretación de los resultados, revisión final del manuscrito. 